\title{
NON-TRIVIAL BEHAVIOUR OF THE SCATTERING AMPLITUDE OF CONTACT-INTERACTING ANYONS
}

\author{
Paola Giacconi ${ }^{a, 1}$, Fabio Maltoni ${ }^{b, 2}$, Roberto Soldati ${ }^{a, 3}$ \\ a Dipartimento di Fisica "A. Righi" and Sezione I.N.F.N., \\ via Irnerio 46, 40126 Bologna, Italy \\ ${ }^{b}$ Dipartimento di Fisica and Sezione I.N.F.N., \\ via F. Buonarroti, 56100 Pisa, Italy \\ and TH Division, CERN, Geneva, Switzerland
}

\begin{abstract}
It is shown that the scattering amplitude for contact-interacting anyons does exhibit a genuine non-perturbative sector. This means that the corresponding perturbative field theoretical formulation, based on the $2+1$ non-relativistic Chern-Simons gauge model coupled to self-interacting complex scalar field, is not generally able to reproduce, order by order in perturbation theory, the exact result. It is proven that the full agreement between the exact scattering amplitude and the resummation of the perturbative expansion of the renormalized 1PI amplitude actually occurs only for some continuous sub-family of selfadjoint extensions of the quantum Hamiltonians, which entail the absence of bound states. A comparison with previously obtained results is carefully worked out.
\end{abstract}

PACS numbers : 03.65.Bz, 03.65.Nk, 11.10.Kk, 11.25.D

1 E-mail address: giacconi@bologna.infn.it

2 E-mail address: fabio.maltoni@cern.ch

3 E-mail address: soldati@bologna.infn.it 
I. It is well known since twenty years [1] that the Born approximation fails to reproduce the celebrated Aharonov-Bohm scattering amplitude [2]. Later on, several efforts have been put forward in order to attempt to reproduce the above amplitude, as well as various anyons characteristic features, by means of perturbative power series expansions [3]. There are also recent proposals in the Literature [4-9] to relate, order by order in perturbation theory, the strength of the point-like interaction, for two particles on the plane, with the renormalized coupling of some $2+1$ dimensional non-relativistic field theoretic models. Here we want to show how that issue appears indeed to exhibit remarkable subtleties; moreover we shall be able to point out to what extent the above matter holds true and to compare with previous results.

A first goal [4] was to put into a close relationship the quantum mechanical scattering amplitude, for two particles interacting via $\delta$-like potential on the plane, with the renormalized 1PI four-point function of a self-interacting non-relativistic complex scalar field model. Now, it is well known [10] that $\delta$-like potentials on the plane are not mathematically properly defined at the quantum level. The correct quantum mechanical formalism to describe point-like interactions of two identical point particles with mass $m$ is only in terms of the self-adjoint extensions of the relative Hamiltonian $H_{0}=\left(\mathbf{p}^{2} / m\right)$. This framework will be referred to in the sequel as contact-interaction.

This leads [10] to the non-trivial scattering amplitude - which is entirely due to the $S$-partial wave - given by

$$
\begin{aligned}
\sqrt{\pi i p} f\left(p, E_{0}\right) & \equiv e^{2 i \delta_{0}}-1=\exp \left\{2 i \arctan \frac{\pi}{\ln \left(-p^{2} / m E_{0}\right)}\right\}-1 \\
& =\frac{2 \pi i}{\ln \left(-p^{2} / m E_{0}\right)-i \pi} ;
\end{aligned}
$$

here $p$ is the modulus of the relative momentum, while $E_{0}=E_{B}<0$ is the bound state energy and/or the opposite of the resonance's energy $E_{\mathrm{res}}=-E_{0}$, which labels the one-parameter continuous family of the self-adjoint extensions of $H_{0}$ disclosing, thereby, the presence of some non-trivial attractive interaction due to the boundary condition at the origin. As a matter of fact, it turns out that, for any given non-trivial behaviour at the origin of the square integrable wave function, a bound state always exists [10] which specifies, in the most natural and physical manner, the corresponding self-adjoint extension of $H_{0}$. Notice that the limiting case $E_{B} \rightarrow-\infty$ - the so called Friedrichs' extension corresponding to regular wave functions - leads to a vanishing amplitude, i.e. to the switching off of the contact-interaction (free particle). Consequently, we can conclude that in two spatial dimensions the contact-interaction, which leads to non-trivial physical effects, turns out always to be of attractive nature.

Now, one can try rather easily to identify the quantum mechanical momentum scale $\sqrt{m\left|E_{B}\right|}$, due to the presence of contact-interaction, with the subtraction point $\mu$ of a renormalizable field theoretic model. The question is, therefore, to carefully verify whether such an identification is actually there, taking into account the close relationship between the quantum mechanical scattering amplitude and the renormalized 1PI four-point function - in the center of mass frame - of a non-relativistic field theoretic model: namely,

$$
\sqrt{\pi i p} f(\varphi, p)=\frac{m}{4} \Gamma_{\mathrm{R}}^{(4)}(p, \varphi),
$$


which holds true in $2+1$ dimensions.

The model is that of a self-interacting complex scalar field, as described by the following renormalized lagrangean density in $2 \omega+1$ dimensions

$$
\mathcal{L}_{R} \equiv \mathcal{L}+\mathcal{L}_{\text {c.t. }}=\phi^{*}\left(i \partial_{t}+\frac{1}{2 m} \triangle\right) \phi-\mu^{2 \epsilon} \frac{\lambda_{0}}{4 m}\left(\phi^{*} \phi\right)^{2}
$$

where $\triangle \equiv \partial^{2}$, the bare coupling $\lambda_{0}$ being determined, order by order in perturbation theory, by

$$
\begin{aligned}
\lambda_{0} & \equiv a_{0}(\lambda)+\sum_{k=1}^{\infty} a_{k}(\lambda) \frac{1}{\epsilon^{k}} \\
& =\lambda+F(\epsilon, m / \mu)+\frac{\lambda^{2}}{8 \pi \epsilon}+\frac{\lambda^{3}}{64 \pi^{2} \epsilon^{2}}+\mathcal{O}\left(\frac{\lambda^{4}}{\epsilon^{3}}\right),
\end{aligned}
$$

$\epsilon \equiv \omega-1$ and $\mu$ is the conventional mass parameter within dimensional regularization. Here, the quantity $F(\epsilon, m / \mu)$ is the usual arbitrary finite part of the counterterm (analytic for $\epsilon \rightarrow 0$ ), which has to be fixed by some renormalization prescription, while all the coefficients of the higher poles $\left(1 / \epsilon^{k}\right)$ can be readily computed from renormalization group equations.

Now, it is an easy exercise to show that, if the finite part of the counterterm for the one-loop 1PI four-point function - which turns out to be the only renormalization part - is chosen to be equal to zero (MS-scheme), then the exact renormalized 1PI four-point function, in two spatial dimensions and in the center of mass frame, reads

$$
\Gamma_{\mathrm{R}}^{(4)}\left(p / \mu^{\prime}\right)=\left(-i \frac{\lambda}{m}\right) \frac{1}{1-(\lambda / 4 \pi)\left\{\ln \left(p / \mu^{\prime}\right)-i(\pi / 2)\right\}},
$$

where $\mu^{\prime}$ is defined from $\ln \mu^{\prime}=\ln \mu+(1 / 2)\left[\ln (4 \pi)-\gamma_{E}\right], \gamma_{E}$ being the Euler-Mascheroni constant. According to Ref. [4], a comparison between Eq.s (1) and (5) allows us to recognize the following correspondences, taking Eq. (2) into account: namely,

$$
\begin{aligned}
\frac{8}{m} \tan \delta_{0}\left(p, E_{B}\right) & =\frac{(8 \pi / m)}{\ln \left(-p^{2} / m E_{B}\right)}=i \Gamma_{\mathrm{R}}^{(4)}\left(i p / \mu^{\prime}\right), \\
\frac{4 \pi}{\lambda} & =\ln \left(\frac{\sqrt{m\left|E_{B}\right|}}{\mu^{\prime}}\right) ;
\end{aligned}
$$

here $\delta_{0}\left(p, E_{B}\right)$ denotes the phase shift associated to the scattering amplitude of Eq. (1). We notice that, owing to the fact that only attractive pure contact-interactions on the plane are non-trivial, i.e. a bound state is always present [4], [10], there is no relationship between the nature of the two-dimensional pure contact-interaction and the sign of the scalar self-interaction, at variance with one- and three-dimensional cases [11]. In other words, the sign of the renormalized coupling $\lambda\left(\mu^{\prime}\right)$ is merely determined by the relative magnitude between the physical scale $\sqrt{-m E_{B}}$ and the definite (but arbitrary) choice 
of the subtraction scale $\mu$, the attractive nature of the contact-interaction being anyway understood.

It is apparent that Eq. (7) precisely provides the above mentioned relationship among the renormalized coupling $\lambda$, within the MS-renormalization prescription, the mass scale $\mu$ and the bound state energy $E_{B}$. It is worthwhile to notice that, from Eq. (7) and taking into account that the bound state energy $E_{B}$ is a physical parameter - i.e. $\mu$-independent - one immediately gets [4] that the $\beta$-function is exactly given by

$$
\beta(\lambda)=\frac{\lambda^{2}}{4 \pi}
$$

This exact value can also be obtained from perturbation theory, to any order, by noticing that there are no higher order corrections - in the running coupling $\lambda\left(\mu^{\prime}\right)$ - to the simple pole $(1 / \epsilon)$ in Eq. (4). Furthermore, it appears that Eq. (5) truly corresponds to the sum of all the renormalized Feynman graphs and turns out to be, as it does, analytic function of the renormalized coupling parameter $\lambda$.

The actual correspondences based upon Eq.s (2) or (6), appear to be firmly established as they stand; it is natural to see, therefore, whether there exists a generalization in the presence of the Aharonov-Bohm interaction, i.e. whether the scattering amplitude for non-relativistic contact-interacting (or colliding) anyons may be exactly reproduced, after resummation of the perturbative series of some suitable field theoretic model [6-9].

II. Let us in fact consider the quantum mechanical scattering amplitude, when the Aharonov-Bohm (AB) gauge potential $A_{j}\left(x_{1}, x_{2}\right)=\alpha \varepsilon_{j k}\left(x_{k} / r^{2}\right), j, k=1,2, \varepsilon_{12}=1, r^{2}=$ $x_{1}^{2}+x_{2}^{2}$, is switched on. We can restrict ourselves to the interval $-1<\alpha<0$ cause, as it is well known, for integer values of $\alpha$ the Aharonov-Bohm quantum Hamiltonian is gauge equivalent to the previously discussed pure contact-interaction Hamiltonian. There, once again, the contact-interaction is described by the self-adjoint extensions ${ }^{\sharp}$ of the relative hamiltonian symmetric operator $H_{\alpha}=(1 / m)[\mathbf{p}-\mathbf{A}(\mathbf{r})]^{2}$.

As we are here interested in the boson scalar like matter, we have to consider the radial differential operator with vanishing angular momentum: namely,

$$
h_{s}(\alpha)=\frac{\hbar^{2}}{m}\left\{-\frac{d^{2}}{d r^{2}}-\frac{1}{r} \frac{d}{d r}+\frac{\alpha^{2}}{r^{2}}\right\} .
$$

The general solution of the eigenvalue equation for the $S$-wave can be written in the form

$$
\psi_{s}(p r)=A J_{|\alpha|}(p r)+B N_{|\alpha|}(p r),
$$

with $p=\sqrt{m E}$ labelling the continuous part of the spectrum, $J_{|\alpha|}(p r)$ and $N_{|\alpha|}(p r)$ being the Bessel and Neumann functions respectively. Notice that the coefficients $A$ and $B$ can always be chosen to be real without loss of generality.

$\sharp$ As the AB hamiltonian operator has deficiency indices $(2,2)$, the most general form of the self-adjoint extensions of the $\mathrm{AB}$ hamiltonian operator is provided by a four parameter family, whose elements do not commute, in general, with the angular momentum operator [12]. 
It turns out [10],[13],[14] that the one parameter continuous family of the self-adjoint extensions of the radial Hamiltonian of zero angular momentum can be described in terms of the quantity $E_{0}$ according to

$$
\gamma \equiv \frac{A}{B} \sin \pi \alpha-\cos \pi \alpha=\operatorname{sgn}\left(E_{0}\right)\left(\frac{p^{2}}{m\left|E_{0}\right|}\right)^{\alpha}
$$

where $-\infty \leq E_{0}<+\infty$, the energy scale $E_{0}$ being here evidently assumed to be independent from the magnetic flux and/or statistical parameter $\alpha$, as it has to label the self-adjoint extensions. In all and only the cases in which $-\infty<E_{0}<0$, there always exists a bound state whose energy is precisely $E_{B}=E_{0}$. Furthermore, the case $E_{0}=-\infty$ corresponds to the original Aharonov-Bohm quantum Hamiltonian [2].

It also happens that the analysis of the stationary scattering states leads to the following $S$-wave phase shift

$$
\tan \left\{\delta_{0}\left(p, \alpha, E_{0}\right)-\frac{\pi}{2} \alpha\right\} \equiv-\frac{B}{A}=\sin \pi|\alpha|\left\{\operatorname{sgn}\left(E_{0}\right)\left(\frac{p^{2}}{m\left|E_{0}\right|}\right)^{\alpha}+\cos \pi|\alpha|\right\}^{-1}
$$

Then, an easy exercise leads to the scattering partial $S$-wave amplitude

$$
\sqrt{\pi i p} f_{0}\left(p, E_{0} ; \alpha\right)=\left(1-e^{i \pi \alpha}\right) \frac{1-\operatorname{sgn}\left(E_{0}\right)\left(p^{2} / m\left|E_{0}\right|\right)^{\alpha}}{\exp \{i \pi \alpha\}+\operatorname{sgn}\left(E_{0}\right)\left(p^{2} / m\left|E_{0}\right|\right)^{\alpha}} .
$$

It is immediate to check that, in the limit $\alpha \rightarrow 0$, the pure contact-interaction amplitude of Eq. (1) is readily recovered from Eq. (14) when $-\infty \leq E_{0}<0$, whereas the expression (14) indeed vanishes in the above limit when $0 \leq E_{0}<+\infty$. $*$

The open question is now to establish whether the above quantum mechanical amplitudes can be exactly reobtained order by order in perturbation theory, taking the general relation of Eq. (2) into account, starting from the field theoretic model described by the renormalized lagrangean density in $2 \omega+1$ dimensions

$$
\mathcal{L}_{R}=\mathcal{L}_{\text {matter }}+\mathcal{L}_{\mathrm{CS}}
$$

corresponding to a non-relativistic charged scalar field interacting with a Chern-Simons gauge field [5]. Here we actually have

$$
\begin{aligned}
& \mathcal{L}_{\text {matter }}=\phi^{*}\left(i \partial_{t}+e A_{0}\right) \phi+\frac{1}{2 m}|i \nabla \phi+e \mathbf{A} \phi|^{2}-\mu^{2 \epsilon} \frac{\lambda_{0}}{4 m}\left(\phi^{*} \phi\right)^{2}, \\
& \mathcal{L}_{\mathrm{CS}}=\frac{\kappa}{2} \varepsilon_{j l} A_{l}\left(\partial_{t} A_{j}-\partial_{j} A_{0}\right)
\end{aligned}
$$

with

$$
\lambda_{0} \equiv a_{0}(\lambda, \nu)+\sum_{k=1}^{\infty} a_{k}(\lambda, \nu) \frac{1}{\epsilon^{k}}, \quad \nu \equiv \frac{e^{2}}{\kappa}=2 \pi \alpha
$$

* We observe that the value $E_{0}=0$ corresponds to a well defined self-adjoint Hamiltonian, whose domain is that one of pure irregular wave functions at the origin. 
To be quite general, we understand that the scalar self-interaction renormalized coupling $\lambda$ and the CS coupling $\nu$, which does not renormalize, are independent free parameters. In so doing, the renormalized lagrangean density (3) is recovered in the limit $\nu \rightarrow 0$.

It is possible to show, from Feynman's rules and the power counting criterion, that (15) is stable under radiative corrections, i.e. there is no need of any further counterterm, but scalar self-interaction, to make Green's functions finite to all orders in perturbation theory [15]. It is important to stress that, within the present model, the scalar self-interacting bare coupling is necessarily generated by the radiative corrections induced from the minimal coupling with the Chern-Simons gauge potential.

Now, starting from the lagrangean (15), the perturbative evaluation of the partial $S$ wave renormalized scattering amplitude leads to, up to the two-loop approximation [5-6] and in the MS-renormalization-scheme,

$$
\begin{aligned}
\left.\Gamma_{\mathrm{R}, s}^{(4)}\left(p / \mu^{\prime}, \nu\right)\right|_{2-\text { loop }} & =-i \frac{\lambda}{m}-\frac{\nu^{2}}{2 m}-\frac{i}{m} \frac{\lambda^{2}-4 \nu^{2}}{4 \pi}\left(\ln \frac{p}{\mu^{\prime}}-i \frac{\pi}{2}\right) \\
& -\frac{i \lambda}{4 \pi m} \frac{\lambda^{2}-4 \nu^{2}}{4 \pi}\left(\ln \frac{p}{\mu^{\prime}}-i \frac{\pi}{2}\right)^{2}+i \frac{\lambda \nu^{2}}{24 m},
\end{aligned}
$$

together with the relationship

$$
\lambda_{0}(\epsilon)=\lambda+\frac{\lambda^{2}-4 \nu^{2}}{8 \pi} \frac{1}{\epsilon}+\frac{\lambda}{8 \pi} \frac{\lambda^{2}-4 \nu^{2}}{8 \pi} \frac{1}{\epsilon^{2}}+\mathcal{O}\left(\frac{1}{\epsilon^{3}}\right) .
$$

First we notice that, once again, owing to the absence of higher order corrections to the coefficient of the simple pole in $(1 / \epsilon)$, the $\beta$-function is exactly provided by the expression

$$
\beta(\lambda)=\frac{\lambda^{2}-4 \nu^{2}}{4 \pi},
$$

which means that perturbative scale invariance occurs at the critical values $\lambda_{\mathrm{cr}}^{( \pm)}= \pm 2 \nu=$ $\pm 4 \pi \alpha$, according to Ref. [5]. It should be stressed that those critical values are obtained within the framework of field theoretic perturbation theory; on the other hand, we recall that the exact quantum mechanical amplitude of Eq. (14) exhibits non-perturbative scale invariance if and only if $E_{0}=-\infty, E_{0}=0$.

Now the key point. A straightforward comparison between the quantum mechanical amplitude of Eq. (14) and the field theoretical perturbative expansion of Eq.s (18-19), allows us to establish the domain in the parameter space $(\alpha, \lambda)$ in which the two formulations appear to be exactly equivalent. This analysis shows that the exact correspondence takes place only in the following two cases:

a) $\alpha=0, \forall \lambda \in \mathbf{R}$ (pure contact-interaction);

b) $\lambda=0, \forall \alpha \in]-1,0]$ (CS minimal coupling), provided $0<E_{0}<+\infty$.

Let us in fact consider the limit $\alpha \rightarrow 0$ of Eq. (18); then the expansion in powers of $\lambda$ of the pure contact-interaction amplitude of Eq. (5) is correctly recovered. If, instead, we let $\lambda \rightarrow 0$, then the expansion in powers of $\alpha$ of Eq. (14) is indeed obtained only 
in the domain $0<E_{0}<+\infty$, which is characterized by the absence of bound states. This means that, strictly speaking, only the amplitudes corresponding to the sub-family of self-adjoint Hamiltonians with purely continuous spectrum and non-trivial scaling behavior are actually reproduced by the renormalized minimally coupled Chern-Simons perturbative field theory in the MS-scheme, i.e. with $a_{0}(\lambda, \nu)=0$ and $a_{k}=a_{k}(\nu)$, provided we identify $\mu^{\prime}=\sqrt{m E_{0}}$.

On the contrary, the expansion in powers of $\alpha$ - the strength of the AB interaction of the exact quantum mechanical amplitude (14) in the attractive domain $E_{B}=E_{0}<0$, where a bound state is always present, reads

$$
\begin{aligned}
& \sqrt{\pi i p} f_{0}\left(p, E_{B} ; \alpha\right)= \\
& \frac{(i m / 4) T\left(p, E_{B}\right)}{1-(i m / 8) T\left(p, E_{B}\right)}+\frac{4 i \pi^{2} \alpha^{2}}{3 m T\left(p, E_{B}\right)} \frac{1+(i m / 8) T\left(p, E_{B}\right)}{1-(i m / 8) T\left(p, E_{B}\right)}+\mathcal{O}\left(\alpha^{4}\right),
\end{aligned}
$$

where

$$
T\left(p, E_{B}\right) \equiv \frac{(8 \pi / m)}{\ln \left(-p^{2} / m E_{B}\right)} .
$$

Now, since the first term in the RHS of the expansion is just $\sqrt{\pi i p} f_{0}\left(p, E_{B} ; \alpha=0\right)$, $i$. e. the amplitude of Eq. (1), it follows from Eq. (5-7) that the quantity $T\left(p, E_{B}\right)$ must be given by

$$
T\left(p, E_{B}\right)=\frac{(\lambda / m)}{1-(\lambda / 4 \pi) \ln \left(p / \mu^{\prime}\right)}, \quad E_{B}=-\frac{\mu^{\prime 2}}{m} \exp \left\{\frac{8 \pi}{\lambda}\right\} .
$$

As a consequence, it is absolutely manifest that the coefficient of $\alpha^{2}$ in Eq. (21) is not analytic in the renormalized coupling $\lambda$. Thereby, the resummation of the perturbative expansion of $\Gamma_{\mathrm{R}, s}^{(4)}\left(p / \mu^{\prime}, \alpha\right)$, whose two-loop value is provided by Eq. (18), will never be such to fully reproduce the exact formula (14), which turns out to contain truly non-perturbative effects (the AB effect in the presence of the attractive contact-interaction).

We stress once again that the latter conclusions lies on the assumed independence from $\alpha$ of the energy scale $E_{0}$, which labels the one-parameter family of self-adjoint Hamiltonians. On the other hand, this very same assumption is mandatory in order to correctly reproduce, in the limit $\alpha \rightarrow 0$, the pure contact-interaction amplitude of Eq. (1). An alternative possibility has been considered in Ref.s [6], [7], [8], where it is instead tacitly assumed that the contact-interaction disappears after the switching off of the AB interaction, as we shall further discuss in the sequel.

To sum up, in the parameter plane $(\alpha, \lambda)$ the precise correspondence between perturbative quantum field theory and non-perturbative quantum mechanics is rigorously established only on the coordinate's axes $\alpha=0, \lambda=0$ and only for the continuous sub-family of self-adjoint extensions which do not admit bound states, namely $0<E_{0}<+\infty$.

III. It is very instructive to compare our result with the treatments given in the recent Literature [5-9] and to carefully discuss why different points of view and conclusions do indeed arise.

In order to do this, let us first briefly recollect and comment some of the most frequently used parametrizations and conventions concerning the self-adjoint extensions of 
the Aharonov-Bohm relative radial hamiltonian operator of vanishing angular momentum (see Eq. (10)). Those extensions have to be encoded into the quantity (see Eq.s (11) and $(12))$

$$
\gamma \equiv \frac{A}{B} \sin \pi \alpha-\cos \pi \alpha
$$

The condition for the absence or presence of a bound state, $\forall \alpha \in]-1,0]$, reads respectively: $\gamma=-\infty, \gamma \geq 0$ (absence) and $-\infty<\gamma<0$ (presence).

As the phase shift of the $S$-partial wave is provided by

$$
\tan \left\{\delta_{0}+\frac{\pi}{2}|\alpha|\right\}=-\frac{B}{A}=\frac{\sin \pi|\alpha|}{\cos \pi|\alpha|+\gamma},
$$

the $S$-partial wave scattering amplitude can be immediately written in the form

$$
\sqrt{\pi i p} f_{0}(\alpha ;[\gamma])=\left(1-e^{-i \pi|\alpha|}\right) \frac{1-\gamma}{e^{-i \pi|\alpha|}+\gamma}
$$

Notice that the limit $\gamma \rightarrow-\infty$ corresponds to the original AB amplitude [2].

In order to exhibit some explicit formula for the scattering amplitude, it is mandatory to specify the quantity $\gamma$ as a function of the following independent variables: the modulus $p$ of the center of mass momentum, the magnetic flux (or statistical) parameter $\alpha$ and some further parameter, which labels the continuous family of self-adjoint extensions, which necessarily involves the presence of an additional momentum scale (or length scale). To this aim, two alternative basic choices are available in the Literature: let us briefly analyse both of them.

The first possibility is the one we have previously introduced (see Eq. (12)), which is equivalent to the one of Ref. [14]: namely,

$$
\gamma\left(\alpha, E_{0}\right)=\operatorname{sgn}\left(E_{0}\right)\left(\frac{\sqrt{m\left|E_{0}\right|}}{p}\right)^{2|\alpha|}
$$

with $-\infty \leq E_{0}<+\infty$ and $E_{0}$ is understood to be $\alpha$-independent. We notice that such an energy scale is a physical observable quantity: it represents the resonance energy (when $E_{0}>0$ and $-1<\alpha<-\frac{1}{2}$ ), or the slope of the cross section (when $E_{0}>0$ and $-\frac{1}{2}<\alpha<$ 0 ), or the bound state energy (when $E_{0}<0$ ).

The second possibility is the parametrization used in Ref. [6]: namely,

$$
\gamma(\alpha, w)=\frac{1}{w}\left(\frac{2 \hbar}{p R}\right)^{2|\alpha|} \frac{\Gamma(1+|\alpha|)}{\Gamma(1-|\alpha|)},
$$

with $R$ is some fixed length (say $1 \mathrm{~cm}$ ) and $w \in \mathbf{R}$ independent from $|\alpha|$. Here, the dimensionless quantity $w$ is related to the behavior of the wave function at the origin and, thereby, it does not represent a directly observable quantity.

Now, it is absolutely crucial to gather the following subtle point. For a given fixed nonzero value of the magnetic flux (or statistical parameter) $\alpha$, Eq.s (26) and (27) give perfectly 
equivalent labelling of the continuous one-parameter family of self-adjoint extensions of the $S$-wave radial hamiltonian, according to Von Neumann's theorem.

But, if we want to establish some correspondence with perturbative quantum field theory, we need more, as we must look at the scattering amplitude as analytic function of the variable $\alpha \in]-1,0]$. In so doing, it will be clear, as we shall explain below, that the two options of Eq.s (26) and (27) actually describe physically different situations and, not surprisingly, they lead to quite different conclusions concerning the possibility to fully reproduce the scattering amplitude within perturbative quantum field theory.

i) If we restrict ourselves to the continuous sub-family of self-adjoint extensions labelled by $0<\gamma<+\infty$, it is easy to realize that both parametrizations lead to equivalent descriptions in the following sense. The pure CS model $\lambda=0, \mu^{\prime}=\sqrt{m E_{0}}$ or such an $\alpha$-dependent renormalized scalar coupling

$$
\lambda(\alpha)=4 \pi|\alpha| \frac{1-w}{1+w}, \quad \mu^{\prime}=\sqrt{4 \pi}(2 \hbar / R) \exp \left\{-(3 / 2) \gamma_{E}\right\}
$$

as proposed in Ref. [6], both reproduce, order-by-order in the renormalized couplings, the very same amplitude (25), when $0<\gamma<+\infty$ that means $w>0$, up to some one-to-one redefinition of the parameters. Notice that, if $0<\gamma<+\infty$, the amplitude vanishes when $\alpha \rightarrow 0$, i.e. when the CS-AB interaction is turned off.

ii) On the contrary, within the complementary continuous sub-family of self-adjoint extensions labelled by $-\infty \leq \gamma \leq 0$, that includes the standard AB case, the two parametrizations describe truly different physical situations. As a matter of fact, according to our choice (26), the bound state energy $E_{0}=E_{B}<0$ - a physical observable quantity - is assumed to be independent from the magnetic flux $\alpha$ and, thereby, it becomes the natural parameter which labels the self-adjoint extensions.

Owing to this choice (26), on the one hand the amplitude (21) is not analytic function of the renormalized scalar self-interaction renormalized coupling $\lambda$ but, on the other hand, from the very same choice (26), one does correctly reproduce the non-vanishing pure contact-interaction case of Eq. (1), when $\alpha \rightarrow 0$.

We stress once again that, in so doing, the underlying CS gauge field theory involves a renormalized scalar self-interaction $\lambda$ truly independent from the scalar-CS minimal coupling $\nu$ - which does not renormalize - as it must be on general ground: namely, $\lambda(\alpha=0) \neq 0$ at variance with Eq. (28).

Alternatively, if the amplitude (25) is expressed by means of Eq. (27) with $-\infty<$ $w<0$, then the bound state energy reads:

$$
E_{B}(\alpha, w)=-\left(-\frac{1}{w}\right)^{1 /|\alpha|}\left(\frac{4 \hbar^{2}}{m R^{2}}\right)\left[\frac{\Gamma(1+|\alpha|)}{\Gamma(1-|\alpha|)}\right]^{1 /|\alpha|}
$$

Now, it is quite clear that, under the assumption that $w$ is $\alpha$-independent - what is explicitly done in Ref.s [6] and [9] - the bound state energy, which is a physical quantity, becomes $\alpha$-dependent, at variance with what happens in the previous approach: this is the reason why it can no longer be used, in this approach, as an independent parameter to label the continuous family of self-adjoint extensions. Furthermore, in the limit $\alpha \rightarrow 0$ the bound 
state energy (29) goes to $-\infty$, which means that the pure contact-interaction case can no longer be recovered. Nevertheless it is remarkable that, thanks to Eq. (28), the sub-family of the scattering amplitudes which entail the presence of a bound state (i.e. $-\infty<w<0$ ) might be reobtained after resummation of the perturbative field-theoretic expansion, but for the singular case $w=-1$. It would be very interesting to verify whether the latter circumstance still occurs in the $N$-particle sectors (with $N>2$ ) [16], in the presence of additional interactions other than the $\mathrm{AB}$ potential and in the relativistic case [17].

To sum up, we can draw the following conclusions:

i) in the absence of bound states, the exact solution of the quantum mechanical twobody problem can always be reobtained after resummation of the perturbative expansion from the Chern-Simons field theoretic model in the non-relativistic case.

ii) in the presence of bound states, two alternative situations do occur:

a) if the physical bound state energy $E_{B}$ results to be independent from the magnetic flux (or statistical) parameter $\alpha$, then the renormalized coupling $\lambda$ is also $\alpha$-independent and the scattering amplitude is not analytic in $\lambda$, albeit the pure contact-interacting case is correctly recovered in the limit $\alpha \rightarrow 0$;

b) if the physical bound state energy $E_{B}$ results to be some suitable function of $\alpha$, then the renormalized coupling $\lambda$ is also $\alpha$-dependent and the exact quantum mechanical scattering amplitude can be reobtained after resummation from the CS field theoretic perturbative

approach, but for the special case $E_{B}(\alpha, w=-1)=-\left(\frac{4 \hbar^{2}}{m R^{2}}\right)\left[\frac{\Gamma(1+|\alpha|)}{\Gamma(1-|\alpha|)}\right]^{1 /|\alpha|} ;$ however, the pure contact-interacting case cannot be recovered in the limit $\alpha \rightarrow 0$.

As a final comment, we can say that, since the bound state energy $E_{B}$ and the magnetic flux $\alpha$ are observable quantities, their actual relationship can be in principle experimentally checked. Therefore, the above discussed alternative possibilities of describing the model in terms of perturbative quantum field theory do eventually concern the concrete physical framework to which the model itself could actually be applied.

\section{Acknowledgments}

This work is partially supported by a grant MURST - quota 40\%. We thank the CERN Theory Division for the kind hospitality during the last stage of the work. We wish to thank R. Adami for helpful discussions and D. Bak for useful correspondence. One of the authors (R. S.) received great benefits from conversations with M. Consoli, C. Manuel, S. Korenblit, V. Leviant, D. Naumov, S. Ouvry and R. Tarrach. We are specially indebited to G. Amelino-Camelia for his stimulating contribution to improve a previous version of the present paper.

\section{References}

[1] E. Corinaldesi, F. Rafeli : Am. J. Phys. 46 (1978) 1185.

[2] Y. Aharonov, D. Bohm : Phys. Rev. 115 (1959) 485.

[3] C. Chou : Phys. Rev. D 44 (1991) 2533;

G. Amelino-Camelia: Phys. Lett. 286B (1992) 97;

S. Ouvry : Phys. Rev. D51 (1994) 5296;

C. Manuel, R. Tarrach : Phys. Lett. 328B (1994) 113; 
G. Amelino-Camelia : Phys. Rev. D51 (1995) 2000.

[4] O. Bergmann : Phys. Rev. D46 (1992) 5474.

[5] O. Bergmann, G. Lozano : Ann. Phys. (N.Y.) 229 (1994) 416.

[6] G. Amelino-Camelia, D. Bak : Phys. Lett. 343B (1995) 231.

[7] S.-J. Kim : Phys. Lett. 343B (1995) 244.

[8] D. Bak, O. Bergmann : Phys. Rev. D51 (1995) 1994.

[9] S.-J. Kim, C. Lee : Phys. Rev. D55 (1997) 2227.

[10] S. Albeverio, F. Gesztesy, R. Hoegh-Krohn, H. Holden : "Solvable Models in Quantum Mechanics" (Springer-Verlag, New York, 1988).

[11] R. Jackiw, in M.A.B. Beg Memorial Volume, A. Ali and P. Hoodbhoy Eds. (World Scientific, Singapore, 1991).

[12] R. Adami, A. Teta : preprint 97/4, Dip.to di Matematica, Roma "La Sapienza" (1997).

[13] P. Giacconi, F. Maltoni, R. Soldati : Phys. Rev. D53 (1996) 952.

[14] C. Manuel, R. Tarrach : Phys. Lett. 268B (1991) 222.

[15] D. Z. Freedman, G. Lozano, N. Rius : Phys. Rev. D49 (1994) 1054.

[16] A. N. Vall, S. E. Korenblit, V. M. Leviant, D. V. Naumov, A. V. Sinitskaya : hepth/9710108.

[17] K. Chadan, N. N. Khuri, A. Martin, T. T. Wu : hep-th/9805036. 